\title{
Research on Lateral Transfer Audio-Visual Teaching based on Corpus Qin $\operatorname{Bin}^{1, a}$ \\ ${ }^{1}$ School of Foreign Language, Neijiang Normal University, Neijiang, Sichuan, China, 641112 ${ }^{a}$ email,
}

\author{
Keywords: Audio-Visual Teaching, Learning Transfer, Corpus, Teaching Strategy
}

\begin{abstract}
Mobility is a common thinking phenomenon of learning and it also plays an important role in the learning process. In the audio-visual teaching based on the corpus, the learners, corpus and teachers' audio-visual teaching have direct or indirect effects on transfer. The paper firstly analysis the main transfer theory of teaching, and then learning corpora was constructed on this basis, corpus and other teaching and teacher-visual teaching are analyzed in order to provide a useful reference for the development of foreign language education.
\end{abstract}

\section{Introduction}

Transfer is a common phenomenon in human learning, the impact of individual learning in a situation of learning in the context of another. In the field of educational psychology, research on the transfer of both theory and practice of great significance, not only helps to reveal the nature and the laws of learning, deepen and expand research study psychology, but also to make students have learned can be carried out reproduction and recycling, and promote innovation. "Teaching for Transfer" has become the consensus of educators, in foreign language teaching, science teaching is the core of the transfer scenario, in which scenario the "source" is taken from the scene corpus, the transfer tool is electronic teaching. Visible, electronic teaching based transfer scenarios corpus has important research value. Based on the above thinking, this paper clarify Language Teaching lateral transfer theories and methods and the theoretical basis of the French electronic teaching, for example, to build the French scene corpus, and supporting scientific electronic teaching means to enhance foreign language teaching effectiveness, and can be replicated form of standardized electronic teaching mode.

\section{The Theoretical Summary}

Transfer learning theory has been throughout human learning process. The theory dates back to the Spring and Autumn Period, Confucius's "art corner, not with three corner against" the teaching point of view is the prototype of contemporary transfer Thought. Thought overseas transfer was first formed in Plato and Aristotle. After the British educator John Locke on learning the Transport phenomenon summarized, systematically put forward the "transfer" concept. Faculty psychology-based training in the form of transfer issues, said school learning to make attribution, functional proposition can be formed by a corresponding development of the training, if the previous study and subsequent study involving the same functional, it will enhance the production of subsequent learning role, showing the transfer phenomenon. Early 20th century, in the form of training say challenged, James (1890) Through Poetry "," Forest Goddess "memory" learning transfer research experiments in the form of training, said negative transfer theory point of view, although he did not summarize the new transfer theory, but this experiment will improve performance under certain cognitive strategies of the last time the same cognitive strategies have a positive impact on the conclusions; Thorndike (1903) by estimating the area experiments that the same functional not necessarily occur transfer phenomenon, and proposed a "common elements, said," that is, only when there is the same between the two elements of the training function, a function of the change in order to change the function of another acquisition. Judd (1908) in the "common elements, said," based on the "bottom targeting" experiment, that "In summary," the transfer theory, the theory that relates to the common principles of the two learning, must have a 
"common element "transfer can occur, mainly due to transfer occurs study, is to learn the main principles of the common study-related situations summarized. Le harsh (1929) based on "chick food" experiment, that "the relationship between the conversion say" transfer theory that transfer is key subjects of context relationships (or gestalt) epiphany, if you have two problems the same deep structure of relationship, a problem for which the training will have to migrate to another problem. Aosigute (1949) comprehensive study of dual learning, that "three-dimensional surface plot Transfer" and it thinks that different combinations of the similarity of the two dimensions of the stimulus and response generation learning transfer effect, which reflects to some extent, "said the common elements "deepening. Then, with the rise of cognitive psychology, cognitive thought penetrated into the field of educational psychology. Ausubel (1978) First, from the cognitive perspective on transfer issues were clearly discussed, he thought cognitive structure is an important factor to be generated by the transfer, the original cognitive structure clarity, stability, generality, inclusive organizational characteristics, coherence and legibility etc. always affects new learning is obtained and maintained. On this basis, Bassok \& Holyoak (1990) proposed a symbolic schema (symbolic schema) theory, which advocates abstract drawings (structural characteristics) and special role to play specific content (surface features) during the transfer have played a certain effect. Subsequently, Sternberg \& Frensch (1993) proposed transfer depending on the coding of the special nature of the information to identify the organization and yearned mechanisms. Therefore, the transfer of cognitive-oriented research is "based transfer Memory" (Memory-Based Transfer) (Pyhe, 1990), or "schema transfer based" (Schema-Based Transfer), as Gray and Orasanu said "learner mental model itself is learning to migrate." These views emphasis on the individual minds of cognitive schemata factors influence transfer, reflecting the transfer of top-down, that transfer occurred starting in the minds of the general knowledge about learning objects, use this knowledge to guide the transfer is restricting a high level of processing procedure of a lower level of processing.

The late 1980s, situational learning theory (Situated Theories) the emergence of researchers for transfer factors concern the construction of cognitive orientation into alignment. Constructivism believes that the situation should be characterized as a factor to consider transfer and transfer is based on the context of predisposing factors. Cognitive schema transfer is not necessarily required, in the absence of a detailed description of the property symbolic representation context and the context of the stimuli can also be perceived. Learning needs in the real physical and social situations occur in the future so that learners can use the context of knowledge and skills. Thus, with the transformation of research oriented to Greeno (1993) as the representative of the researchers thus proposed transfer situational theory that transfer is to specify in a situation in which learning how certain activities affect other context of another activity. When learners findings into the context containing the original context of providing the same or similar composition, will migrate occur. The establishment of such activities depends both on the structure of the initial learning situation, and subject to its subsequent transfer scenarios. The role of context is to induce and provide specific activities. Through interaction with the individual links in the event of the formation of the dynamic activity diagram type, tissue type that is the principle activity diagram activity. Transfer activity is to use drawings to adapt to different situations. Other studies have demonstrated that the strengthening of social activities and cooperative learning, and form practical inference schema (Pragmatic Reasoning Schemas), promote the occurrence of transfer. This schema does not rely on abstract symbolic drawings, but instead relies on the knowledge and associated context, including specific information about specific events or situations and specific experience in the case. Even many experts also pointed out that unless it is in their own areas of expertise, generally do not use or rarely use abstract symbols drawings. In summary, based on Learning Transfer of situated learning theory is a positive breakthrough in the conventional view, pointing out the characteristics of the transfer situation. Therefore, from the perspective of context to discuss transfer issues will undoubtedly help to reveal the mechanism of transfer occurred, deepening and expansion of transfer studies.

With the continuous development of computer technology, linguistics and computer science were 
combine and empirical analysis by multi-angle multi-level, and promote the continuous development of foreign language corpus. By constructing scenarios corpus, and the use of modern advanced electronic teaching means, through a similar scenario comparison study, based on a lateral transfer learning theory scientific and rational design of teaching paradigm to maximize the release of corpus resources to enhance the learning efficiency of learning the subject.

\section{Transfer Study of Learning Subject Audio-Visual Teaching Schema}

Learning transfer is not spontaneous, but subject to a number of factors. In Foreign Language Education, the education, the educated and constitute the basic elements of education on teaching activities. This article from the main body of learning, teachers teaching as electrical power and similar scenarios establishing three dimensions of Foreign Language Education to study these three parts is a necessary factor in teaching activities, are indispensable and complementary.

Learners often encounter with two questions "listening" and "say": I can understand too little; I know what I want to express, but do not know what words more appropriate. And the teaching process, we found, each learner will encounter the same Western Corpus transfer learning to improve foreign language teaching. In the process of building a corpus, we need to take into account the mobility of foreign language learners' cognitive structure characteristics, generalization and emotional factors in three areas.

Learning cognitive structure subject to subsequent learning has a greater impact. Foreign Language Teaching involved and learning scenarios tend to be the main past cognitive structure, knowledge and experience linked together, which is now learning into learners existing cognitive structures. Bin Qin (2016) considered good cognitive structure based on knowledge and experience to quickly and accurately integrate city obvious correlation between new knowledge, new knowledge in a certain extent, improve the body's own cognitive learning system, both complement each other.

That transfer is summed up and the cognitive structure requires a high degree of generalization. The key according to generalize the theory of transfer theory, learners generate transfer is whether generalize from two learning around the common elements. Western Corpus by summing up before and after the study summarized. The mindset often exhibit a tendency of thinking, which requires corpus lateral transfer process of teaching scenarios repeatedly stressed the need to form a profile that can be replicated, while guiding learners to distinguish situations in order to reduce the negative transference occurs.

Language Learning Corpus objective reality, but the education process of learners' emotional factors has a strong initiative. To study the efficiency of the body's largest acquisition scenario experience, it is necessary to make learning activities become lively, active and full of personality. Experience shows that learning a subject at ambient atmosphere of equality, harmonious, democratic, and it is able to build trust and respect the feelings of the mechanism to reflect the activities of interaction, cooperation, and actively participate in classroom learning, which greatly enhance the learning efficiency. So, in summary of the transfer process of learning, trial and error to allow learning topics, this aspect can improve the corpus and on the other hand can grasp the theme of learning problems learning accuracy.

\section{The Construction of Scenarios Corpus and Audio-Visual Teaching}

Previously characterized similarity learning and subsequent learning math problems between transfers will directly affect the solver occurred and occurrence of negative transfer. Analog Transfer called "example theory" that the previous examples in analogy learning process transfer often has a decisive role. Sample study focused on summarizing the abstract knowledge of this type, in order to achieve the effect of analogical transfer from specific cases. Western scientific and rational design of similar scenarios corpus become electronic teaching an important research topic. Our scholars have conducted a lot of research, the results of most of that in the same class Western scenario, the similarity problem representation of an important impact on transfer issues based on 
analogies to characterize transfer is one of the common themes of problem solving strategies. As can be seen, electrochemical transfer teaching at Western Corpus is particularly important. Scientific and rational design scene corpus can be more prone to transfer analogy between similar scenario, "the source scenario" (already familiar with similar situations) and "target scenario" (a new profile). If designed properly between corpuses, "the source scene" and "target scenario" Because scenario is not obvious surface features, not prone to transfer analogy, and even interfere with the correct direction of transfer.

Taking into account the perspective of electronic teaching scenarios start corpus must be real, unprocessed raw corpus, and can be updated in real time. So this collection of student writing electronic documentation, videos and scenarios scenario describes the dialogue of audio data, create scenarios corpus, and utilization of information and tools corpus provided for learners scenario transfer study for evaluation and analysis, evaluation of foreign language learning ability. French corpus is divided into three parts: the distribution of the scene, the scene corpus body, computer information storage and indexing.

The Distribution of the Scenes. Corpus stored in the actual use of language in real appeared language materials, so the scene must come from the reality of the real scene, select "Reflets", "Concise French Course", "University French", "alter ego", " new without borders "in the scene based on the establishment of a similar scene shard by consolidating clustering, and build a library of convergence between the various sub-libraries.

The Establishment of Scenarios Corpus. Scenario corpus statistical sources include student writing electronic documents, trial and error scenarios described video and audio dialogue scene three sub-libraries. Trial and error scenarios which describe video and audio sub Dialogue Library Reference "Chinese Students' Spoken English (SECCL)" and "Chinese learners Corpus (CLSC)" to build. Each student essay writing electronic document registration of an independent word document is saved, the scene of trial and error and description of each scene dialogue in a separate document retention MP3 and MP4 respectively. In order to facilitate indexing and statistics, including the name of each document described the scene name, complete with time's name, batch and other content, and coding. As the name "Hotel-1998-Stephanie-E01" document, Hotel for a specific scenario, in 1998 the first 01 batches of documents Stephanie E first semester. Each document can be carried out by application of the "View" or "search" function display viewing.

Computer Information Storage and Indexing. Corpus is a computer language as a carrier carrying basic resources of knowledge, stored in a retrieval computer design quality of the corpus using a greater impact. By writing a computer program to achieve six basic functions Corpus: View scenario distribution; see specific scenarios arrangement; see specific scenario writing and audio and video documents; comparing similar documents; Find keyword filters; statistical processing and analysis of results. The results need to design simple and easy to operate. With the continuous development of network technology, Corpus and micro letter, QQ-mail and other means of communication, and combining the results of the study corpus feedback and suggestions.

\section{Corpus Transfer and Teachers Audio-Visual Teaching}

Corpus linguistic knowledge base that hosts resources, it does not mean the language knowledge, science teachers as electronic teaching resources in order to maximize the use of listening. The accuracy of imparting knowledge is mainly manifested in two aspects of the accuracy of their own knowledge and understanding of the accuracy of methods and classroom language organization and presentation. In addition, the actual teaching process, teachers often targeted instructional design and development of methods based on the actual situation of the students, so not only can lead students to carry out activities, take the initiative to acquire knowledge, but also allows students to develop personality and ability . If the teaching method is unreasonable, easy to make students' thinking is not active, it cannot form a good drawings, and even lead to the existing knowledge and experience with the original knowledge and experience confusion, causing negative transfer. Therefore, in the teaching process, the teacher before teaching and it must learn the situation analysis and teaching analysis. Help students form good cognitive structure, improving transport. 
Authentic materials that need further processing (analysis and processing), in order to become a useful resource potential of electronic teaching corpus to release the maximum extent possible. With the advent of the Internet era of information technology, electronic teaching mostly multi-modal input based on the teaching of modern computer technology and network technology, namely the use of auditory, visual, tactile and other sensory, through language, images, sounds, movements and other means of communication and symbolic resources and comparative analysis of the corpus, to deepen understanding and application scenarios.

Foreign Language Education has been integrated into a more open teaching platform, follow the "people-oriented" teaching philosophy, each learner can through modern education technology and multimedia sharing corpus resources to greatly expand the classroom, change the past, monotonous classroom teaching. In addition, electronic teaching is timely, interactive features, emphasis on students' individualized teaching, learning outcomes through micro letter, QQ-mail and other modern means of communication and real-time feedback, and to develop a standardized document was rich feedback results Corpus. In summary, the corpus provide vivid and clear information, broaden their horizons, shorten the cognitive process, and the aim of teaching different learning according to the subject's personality; electronic teaching create vivid teaching situation, to stimulate students' enthusiasm for learning the subject, teaching content lively and scientific teaching structure to achieve the perfect combination to create a real environment for learning a foreign language learners.

"Student-centered," the power of teaching does not mean that the dominant position is not important teachers, teachers in the use of telephone Corpus teaching process, we need to do: 1 . focus on systematic knowledge and students' ability to generalize. Lateral transfer of electronic teaching process refers indirectly scenarios experience into individual scenes experience, and can be used in the course of practice. In this process, students must pass this one generalization skills to understand the nature of knowledge of the law, so that old and new knowledge to integrate. 2. focus on the establishment of the structure of the corpus scene. Corpus respective profiles are not independent abstract existence of the scenarios in the form of a network structure diagram showing a scenario system, and set the appropriate directory and retrieve information tips that can reveal the inclusion of the scenarios between corpus and Progressive and other relations and improve the ability to learn the subject of transfer. 3. To strengthen the analysis of key scenes. Corpus key scenes can act as a "learning sample" effect. As a sample transfer teaching methods, it provides a problem-solving mode and methods, teachers guide students to understand imitation, so as to solve the type of scenario results.

\section{References}

[1] Bassok M. Transfer of domain-specific problem solving procedures[J]. Journal of experimental psychology: learing, memory and cognition, 1990, 16: 522-533.

[2] SternbergR. J, Frensch P.A. Mechanismsof transfer[A]. InD K Detterman, R JSternberg(Eds. ), Transferon trai:l Intelligence, cognition, and instruction [C]. Norwood, NJ: Ablex, 1993. 25-38

[3] Phye G. D. Inductive problem solving: Schema inducement and memory-based transfer[J]. Journal ofEducational Psychology, 1990, 82: 826-831.

[4] GrayW. D, Orasanu J.M. Transfer of cognitive skills[A]. In CormierS.M. Hagman J.D(Eds. ),Transfer of learning: contemporary research and applications [ C ]. New York: Academic press, 1987, 204.

[5] Greeno J.G, Moore J.L, Smith D.R. Transfer of situated learning[A]. InD K Detterman, R JSternberg (Eds).Transferon trial: Intelligence, cognition and instruction[C]. Norwood, NJ:Ablex, 1993. 99 - 167.

[6] Guan Hong. The evaluation corpus-based French language ability [J] PLA University of Foreign Languages, 2004, (06): 55-58

[7] Li Haiyan, Wu Miao Wang Building of French corpus [J] French study, 2008 (04): 101-105 